\title{
Neural Correlates of Intertemporal Choice In Aggressive Behavior
}

\author{
in press at Aggressive Behavior
}

\author{
David S. Chester ${ }^{1 *}$, Sarah Beth Bell ${ }^{2}$, C. Nathan DeWall ${ }^{2}$, Samuel J. West ${ }^{1}$, Marisabel \\ Romero-Lopez ${ }^{3}$, Adam W. Craig ${ }^{4}$ \\ 1Department of Psychology, Virginia Commonwealth University, USA \\ ${ }^{2}$ Department of Psychology, University of Kentucky, USA \\ ${ }^{3}$ College of Business, Colorado State University, USA \\ ${ }^{4}$ Gatton College of Business and Economics, University of Kentucky, USA
}

Main Text Word Count (minus References, Tables): 4,761

Abstract Word Count: 214

${ }^{*}$ Correspondence should be addressed to:

David S. Chester

Department of Psychology

Virginia Commonwealth University

Richmond, VA, 23284, USA

1-804-828-7624

dschester@vcu.edu 


\section{Acknowledgments}

This work was supported by funds from the University of Kentucky's Gatton College of

Business and Economics (PI: Craig) and by the National Institute on Alcohol Abuse and Alcoholism (NIAAA) of the National Institutes of Health under award number K01AA026647 (PI: Chester).. 


\begin{abstract}
People often have to make decisions between immediate rewards and more long-term goals. Such intertemporal judgments are often investigated in the context of monetary choice or drug use, yet not in regards to aggressive behavior. We combined a novel intertemporal aggression paradigm with functional neuroimaging to examine the role of temporal delay in aggressive behavior and the neural correlates thereof. Sixty-one
\end{abstract} participants (aged 18-22; 37 females) exhibited substantial variability in the extent to which they selected immediate acts of lesser aggression versus delayed acts of greater aggression against a same-sex opponent. Choosing delayed-yet-more-severe aggression was increased by provocation and associated with greater self-control. Preferences for delayed aggression were associated with greater activity in the ventromedial prefrontal cortex (VMPFC) during such choices, and reduced functional connectivity between the VMPFC and brain regions implicated in motor impulsivity. Preferences for immediate aggression were associated with reduced functional connectivity between the VMPFC and the frontoparietal control network. Dispositionallyaggressive participants exhibited reduced VMPFC activity, which partially explained and suppressed their preferences for delayed aggression. Blunted VMPFC activity may thus be a neural mechanism that promotes reactive aggression towards provocateurs among dispositionally-aggressive individuals. These findings demonstrate the utility of an intertemporal framework for investigating aggression and provide further evidence for the similar underlying neurobiology between aggression and other rewarding behaviors. Keywords: aggression, delay discounting, intertemporal choice, $f M R I$, ventromedial prefrontal cortex 


\section{Introduction}

Human life is full of conflict between the allure of immediate rewards and the patience needed to achieve long-term payoffs. Such intertemporal choice dilemmas have been investigated for decades in the context of addiction, monetary investment, and other domains of impulsivity (Berns, Laibson, \& Loewenstein, 2007). However, the psychological and neural factors that influence intertemporal choice in the context of aggressive behavior remain unexamined.

\section{Intertemporal Choice and Delay Discounting: An Overview}

A core component of the literature on intertemporal choice is delay discounting, in which a potential reward gradually loses its value (i.e., is discounted) when associated with a temporal delay (da Matta et al., 2012; Loewenstein, 1988; Reynolds, 2006). People often perceive the subjective value of a delay-associated reward as lower than its objective value. The delay discounting framework posits that when given a choice between an immediate-yet-lesser (i.e., smaller-sooner) reward and a delayedyet-greater (i.e., larger later) reward, the degree of the delay will shift preferences towards immediate-yet-lesser rewards (Kirby, Petry, \& Bickel, 1999).

\section{Neural Mechanisms of Delay Discounting}

The underlying neurobiology of intertemporal choice is complex (see Frost \& McNaughton, 2017). The selection of immediate rewards is associated with activity in the nucleus accumbens, amygdala, posterior cingulate cortex, and anterior insula (McClure, Ericson, Laibson, Loewenstein, \& Cohen, 2007; McClure, Laibson, Loewenstein, \& Cohen, 2004). Conversely, selecting delayed rewards is associated with greater activity in the frontoparietal control network, which serves to override immediate 
impulses for more distal goals (McClure et al., 2004, 2007). Activity in the ventromedial prefrontal cortex (VMPFC) is theorized to represent the subjective value of what is to be gained by selecting the delayed option (Frost \& McNaughton, 2017). Further, effective connectivity between the VMPFC and the dorsolateral PFC (DLPFC) during intertemporal choice is associated with a greater number of delayed reward choices (Hare, Hakimi, \& Rangel, 2014). These findings imply that the selection of delayed rewards, over immediate ones, requires two critical neural ingredients: the representation of the delayed reward's greater value (VMPFC), and the self-regulatory structures necessary to implement the preference (DLPFC; Hare, Camerer, \& Rangel, 2009). Based on the common currency model, such VMPFC-instantiated valuation is similarly computed across different types of rewards (Levy \& Glimcher, 2012). As such, these neural findings that were chiefly based on rewards such as food and money may relate to other sources of reward - perhaps even aggression.

\section{Delay Discounting and Aggression}

There is good reason to view aggression as an intertemporal behavior. Aggression often exists in delayed or impulsive forms (Blair, 2010; cf. Bushman \& Anderson, 2001). For instance, revenge-seekers restrain their retributive impulses, waiting for an opportunity to inflict maximum damage on their victims. Such revengeseekers exhibit a tendency to premeditate their behaviors, suggesting a self-regulated approach to aggression (Chester \& DeWall, 2018). Several questionnaires that assess dispositional aggressiveness include temporally-situated items, such as "I have longliving fantasies of revenge after a conflict is over" and "the more time that passes, the more satisfaction I get from revenge" (Denson, Pedersen, \& Miller, 2006). Conversely, 
some individuals are unable to restrain such impulses and engage in immediate, reactive aggression towards the perceived source of provocation (Raine et al., 2006).

This distinction between delayed and immediate forms of aggression maps onto the well-established constructs of reactive and proactive aggression (Raine et al., 2006). Reactive aggression reflects the tendency to immediately and impulsively aggress towards a perceived source of provocation, whereas proactive aggression is an instrumental, 'cold', and predatory form of aggression that often entails a waiting period between the inception of the act and carrying it out (Raine et al., 2006).

Several studies have tested the relationship between aggression-related constructs and intertemporal preferences for delayed versus immediate rewards. In a laboratory setting, individuals who exhibited more delay discounting also exhibited more psychopathic traits and behaved more aggressively (Miller \& Lynam, 2003). Angry individuals show a preference for immediate over delayed rewards (Zhao, Kirwen, Johnson, \& Vigo, 2017). Extending this effect into the 'real-world', delay discounting has been associated with greater aggressive behavior in female and male parolees, community members, and police officers (Cherek \& Lane, 1999; Cherek, Moeller, Dougherty, \& Rhoades, 1997; Koepfler, Brewster, Stoloff, \& Saville, 2012; Moore \& Foreman-Peck, 2009). These associations implicate intertemporal choice as a likely component of aggressive behavior. Yet why would a reward-based phenomenon play a role in whether individuals act aggressively?

\section{Reward and Aggression}

Aggression is hedonically-rewarding (Chester, 2017). Individuals often seek out the pleasure of aggression in order to maintain affective homeostasis (Chester \& 
DeWall, 2017). This motivation appears in human neurobiology. Aggression behavior has been repeatedly associated with heightened activity in the ventral striatum, a core substrate for feelings of positive affect (Chester \& DeWall, 2016; Chester, Lynam, Milich, \& DeWall, 2018). A major regulatory component of the reward network, the VMPFC, plays a critical role in regulating and constraining aggressive behavior (Blair, 2010).

\section{The VMPFC and Intertemporal Aggression}

The VMPFC plays a central role in successful human self-regulation (Berkman, Livingston, \& Kahn, 2017). It achieves these regulatory ends via robust connections to subcortical regions that are known correlates of reactive aggression (Blair, 2010, 2016; Chester, Lynam, Milich, \& DeWall, 2017). The VMPFC's ability to inhibit reactive aggression may reflect its ability to tune individuals' valuation away from an immediately-gratifying act and towards a more delayed (and more satisfying) outcome (Frost \& McNaughton, 2017). As such, the VMPFC is a likely region that encodes individuals' preferences for immediate versus delayed aggression.

\section{The Present Study}

To assess the nature of intertemporal choice in the context of aggression, we conducted a study that combined functional magnetic resonance imaging (fMRI) with a novel behavioral aggression paradigm. In this paradigm, individuals compete against an opponent to whom they could repeatedly assign an immediate, though lesser, amount of harm, or a delayed, though more severe, amount of harm. Participants completed this task while undergoing $\mathrm{fMRI}$ and then reported their dispositional levels of aggression and self-control to better understand how responses on this task map onto broader 
patterns of individual differences. Based on the common currency model and the role of positive affect in aggression, we predicted that delayed aggression would be associated with greater activity in the VMPFC and DLPFC. Further, we expected that these neural mechanisms of delayed aggression would map onto greater levels of self-control and less overall aggressiveness. Exploratory analyses sought to examine the functional connectivity profiles of these cortical regions during immediate and delayed aggression. Finally, we tested whether profiles of PFC activity during intertemporal aggression decisions served as mechanisms to explain the preference for delayed or immediate aggression among dispositionally-aggressive individuals. To do this, we tested whether PFC activity exhibited an indirect effect between trait physical aggression and decisions on the intertemporal aggression task.

\section{Methods}

\section{Ethics}

All research procedures were approved prior to conducting the study by the appropriate institutional research ethics board, in accordance with United States research ethics guidelines. All participants provided informed consent prior to completing any research procedures, and were fully debriefed as to all deception and true purposes of the study at the end of their participation.

\section{Participants}

Participants were 61 healthy, right-handed, young adults (37 females, 24 males; age: $M=18.98, S D=1.07$, range: $18-22$ ). This sample size was determined based on those employed in previous $\mathrm{fMRI}$ investigations of the neural correlates of aggressive behavior (e.g., Chester \& DeWall, 2016). Participants were undergraduates recruited 
through the introductory psychology subject pool in exchange for credit towards their course's research requirement and a structural image of their brain. Exclusionary criteria was assessed by an online questionnaire, which included: body mass index above 30, claustrophobia, color blindness, mental or neural pathology, metallic objects in the body, prior head trauma, and psychoactive medication use.

\section{Materials}

Brief Aggression Questionnaire. To measure trait physical aggression, we employed the Brief Aggression Questionnaire (BAQ; Webster et al., 2014). The BAQ contained twelve items that comprise four, three-item subscales: anger, hostility, physical aggression, and verbal aggression. Participants responded to each item along a 1 (disagree) to 7 (agree) Likert-type scale.

Brief Self-Control Scale. The BSCS is a 13-item self-report questionnaire developed by Tangney, Baumeister, and Boone (2004) to assess individual differences in trait self-control. Items were rated on a 1 (not at all like me) to 5 (very much like me) response scale.

\section{Procedure}

Pre-scan. Participants arrived at the neuroimaging laboratory where they were told that they would complete a competitive reaction-time task against a same-sex opponent. After practicing the reaction-time task, participants were then placed in an MRI scanner and had a high-resolution structural scan taken of their brain followed by an unrelated functional scan. Then, participants completed our aggression task while undergoing $\mathrm{fMRI}$. 
InterTemporal Aggression Paradigm (ITAP). To assess intertemporal decision-making in the context of aggressive behavior, we adapted the well-established Taylor Aggression Paradigm, which has been effectively implemented in the fMRI environment (Chester \& DeWall, 2016; Krämer, Jansma, Tempelmann, \& Münte, 2007; Taylor, 1967). As in previous iterations of the task, participants repeatedly competed against a same-sex stranger to see who could press a button faster when a prompt appeared. In reality, there was no other person and participants completed the task against a pre-set computer program. As an ostensible motivational component of the task, participants were punished if they lost a given round of competition by receiving an aversive noise blast through a pair of headphones. Conversely, if participants won the round of competition their opponent heard the noise blast and they did not.

In the InterTemporal Aggression Paradigm (ITAP), participants could choose between administering a softer noise blast $(75 \mathrm{db})$ that would be administered in the next few seconds (assuming the participant won that competition), or a much louder noise blast $(105 \mathrm{db})$ that would be administered to the opponent at the end of the study, approximately one hour after the current task was completed (again, assuming the participant won that competition). These larger blasts would be ostensibly administered to the participant's opponent, sequentially, until the number of assigned blasts was reached. To experimentally manipulate provocation within-subjects, participants were assigned both 75db (low provocation) and 105db (high provocation) blasts from their opponent. To ensure that louder blasts would be perceived as more provocative by participants, participants were informed that the noise blasts assigned by their 
opponents would be administered as soon as they lost a given competition, not at a later time in the study.

The task consisted of 26 trials, with each trial containing 6 events (Figure 1). Each trial began with a fixation cross that modeled baseline neural activity (7.5s). Then, participants completed an aggression choice phase in which they selected one of the two aggression options (5s). A blank screen then appeared followed by a red square that indicated to participants that they should press a button as fast as possible to compete against their opponent (5s). Participants then saw what volume level their opponent set for them (5s). Finally, participants saw whether they won or lost the competition and experienced the corresponding noise blast if they lost (2.5s).

Figure 1. Schematic of the InterTemporal Aggression Paradigm.

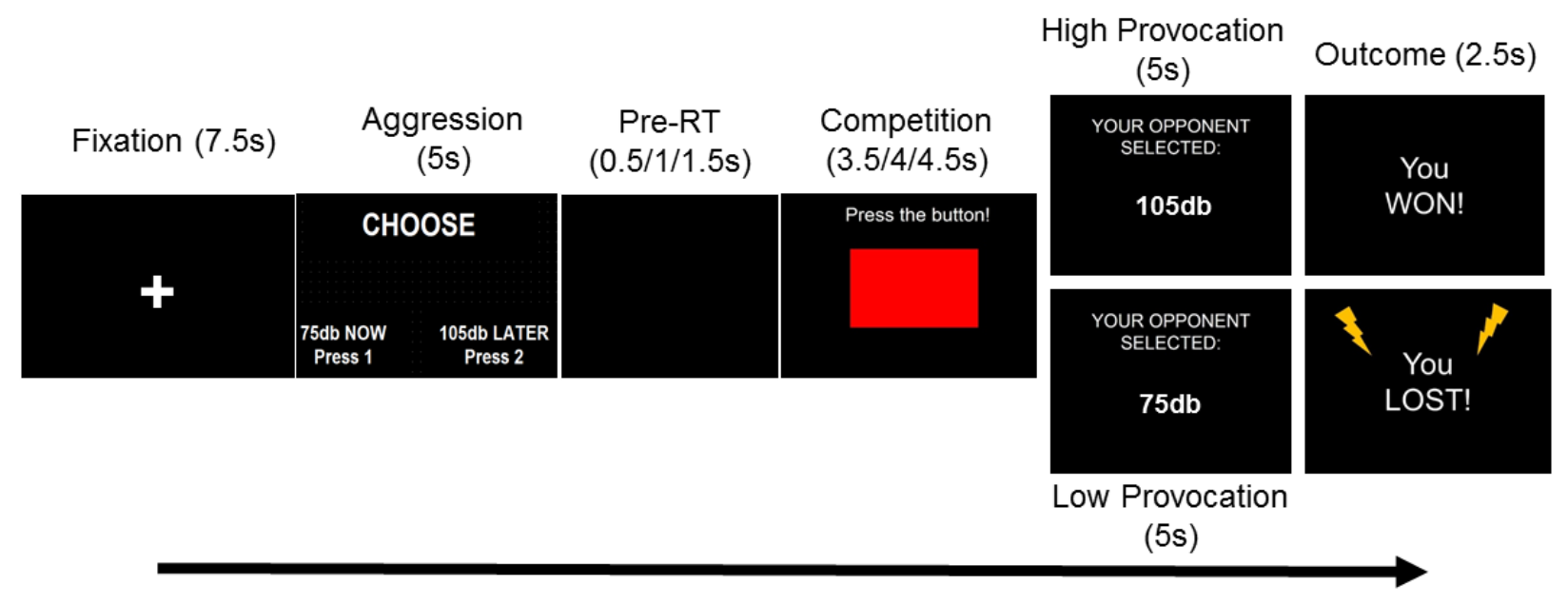

Aggression events were denoted as 'Retaliatory' if the opponent selected $105 \mathrm{db}$ and 'Non-Retaliatory' if the opponent selected $75 \mathrm{db}$ (order of trial-types are listed in Supplemental Table 1). There were 14 Retaliatory trials and 12 Non-Retaliatory trials and there were a greater number of wins (19) than losses (7). The order of provocations 
and non-provocations, as well as wins and losses, were initially randomized and then held constant across participants.

Post-scan. After the aggression task, participants were placed in a nearby testing room and completed a computerized battery of questionnaires including a demographics survey, the Brief Aggression Questionnaire, and the Brief Self-Control Scale. Participants were interviewed as to the success of the fake partner deception, yet participants responses to this interview were not recorded in a systematic way that would allow for an objective determination of deception success.

\section{MRI Data Acquisition and Preprocessing}

All MRI data were obtained using a 3.0 Tesla Siemens Magnetom Trio scanner and a 32-channel headcoil. Echo planar BOLD images were acquired with a T2*weighted gradient across the entire brain with a 3D shim (field of view $=224 \times 224 \mathrm{~mm}^{2}$, echo time $=29 \mathrm{~ms}$, repetition time $=2.5 \mathrm{~s}$, slice thickness $=3.5 \mathrm{~mm}, 40$ interleaved slices, flip angle $=80^{\circ}$ ). To allow for registration to native space, a coplanar, magnetizationprepared rapid gradient-echo (MPRAGE) sequence was also acquired from each participant (field of view $=256 \mathrm{~mm}$, slice thickness $=1 \mathrm{~mm}$, echo time $=2.26 \mathrm{~ms}$, repetition time $=2.53 \mathrm{~s}$, flip angle $=7^{\circ}$ )

The Oxford Center for Functional MRI of the Brain (FMRIB)'s Software Library (FSL version 5.0) was used to conduct all preprocessing and fMRI analyses (Smith et al., 2004; Woolrich et al., 2009). Reconstructed functional volumes underwent head motion correction to the median functional volume using FSL's MCFLIRT tool. FSL's Brain Extraction Tool was used to remove non-brain tissue from all functional and structural volumes using a fractional intensity threshold of 0.5 . After a series of data 
quality checks, functional volumes underwent interleaved slice-timing correction, prewhitening, spatial smoothing (using a 5mm full-width-half-maximum Gaussian kernel), and temporal high-pass filtering (100s cutoff). These processed brain volumes were then fed into subsequent data analyses.

\section{MRI Data Analysis}

Preprocessed fMRI datasets were analyzed using two-level general linear modeling.

First level (within-participants). Each participant's whole-brain functional volumes were entered into a fixed-effects analysis that modeled events using a canonical double-gamma hemodynamic response function with a temporal derivative. Regressors-of-interest for the aggression task included Retaliatory Aggression and Non-Retaliatory Aggression, while leaving fixation events un-modeled. All five seconds of each aggression trial were modeled in this analysis. All other events were included as nuisance regressors. Six head motion parameters from each participant were modeled as nuisance regressors. Linear contrasts compared retaliatory and non-retaliatory aggression to each other (Retaliatory Aggression > Non-Retaliatory Aggression). An additional analysis was conducted that included two regressors for each time participants selected the delayed option and the immediate option, contrasts compared quieter-now to louder-later decisions (Delayed Aggression > Immediate Aggression). Resulting contrast images from these analyses were first linearly registered to native space structural volumes and then spatially normalized to a Montreal Neurological Institute (MNI) stereotaxic space template image (resampled into $2 \times 2 \times 2 \mathrm{~mm}^{3}$ voxels). 
Second level (across-participants). Each participant's contrast volumes from the first level were then fed into FLAME 1's group level, mixed effects GLM that created whole-brain group average maps for each contrast. Cluster-based, family-wise error correction based on Gaussian random field theory was then applied across the entire brain to each of the group activation maps (corrected threshold: $Z>2.3, p<.05$; Heller, Stanley, Yekutieli, Rubin, \& Benjamini, 2006; Worsley, 2001).

For the subset of participants whose behavior on the task was modeled, multiple comparisons corrections were constrained to left and right VMPFC region-of-interest (ROI) masks. Both left- and right-hemisphere VMPFC ROI masks were defined as the medial aspect of the orbitofrontal gyrus, using the Automated Anatomical Labeling atlas (Tzourio-Mazoyer et al., 2002). For entry into subsequent correlational analyses, parameter estimates were extracted from all significant voxels of contrast clusters in units of percent BOLD signal change.

Psychophysiological interaction analysis. To assess functional connectivity during intertemporal choices regarding aggression, psychophysiological interaction (PPI) analyses were planned for the subset of participants included in the previous behavioral analyses. These took the form of first level, within-participants analyses with the addition of two new regressors to the previously described GLM: the mean-centered timecourse of activity in the VMPFC seed region, and an interaction term multiplying the seed region's functional timecourse by delayed aggression decisions. A separate, firstlevel PPI analysis was performed using immediate aggression decisions and their interaction with the VMPFC timecourse. Within each analysis, linear contrasts compared the interaction between the seed region timecourses, aggression decisions, 
and their interaction to participants' implicit baseline. These individual contrast maps were aggregated into a second level, between-subjects analysis, and then family-wise error correction based on Gaussian random field theory was applied across the entire brain (corrected threshold: $Z>2.3, p<.05$ ).

\section{Results}

\section{Behavioral and Self-Report Data: Descriptive Statistics and Zero-Order Correlations}

To quantify intertemporal aggression, participants' selections were coded as 1 ( $75 \mathrm{db}$ now) or 2 (105db later), and then averaged across all 26 trials to create an intertemporal aggression index, whereby higher values indicated more delayed-yetsevere aggression. This index was also separately computed for retaliatory and nonretaliatory trials. On retaliatory events, participants more frequently selected the delayed option than on non-retaliatory events, $t(60)=3.63, p=.001, d=0.47$.

Participants exhibited substantial variability in their intertemporal aggression strategies, as well as in their self-reported trait physical aggression and self-control (Table 1). Participants who more frequently chose the later-louder aggression option were higher in self-control, but not in trait physical aggression (Table 1). Age and gender were unassociated with intertemporal aggression decisions (Table 1). Adequate internal consistency was observed for scores on the overall ITAP, $\alpha=.89$, retaliatory ITAP, $\alpha=.83$, non-retaliatory ITAP, $\alpha=.80$, Brief Aggression Questionnaire - Physical Aggression subscale, $\alpha=.69$, and Brief Self-Control Scale, $\alpha=.89$. 
Table 1. Descriptive statistics and zero-order correlations between behavioral and self-report variables from all 61 participants. Gender coded: $1=$ male, $0=$ female. ${ }^{*} p<.05,{ }^{* *} p<.01$

\begin{tabular}{|c|c|c|c|c|c|c|c|c|c|}
\hline & $M$ & $S D$ & Range & 1 & 2 & 3 & 4 & 5 & 6 \\
\hline 1. Delayed Aggression & 1.22 & 0.22 & $1.00-1.73$ & & & & & & \\
\hline 2. Delayed Aggression - & 1.26 & 0.25 & $1.00-1.79$ & $.96^{* *}$ & & & & & \\
\hline \multicolumn{10}{|l|}{ Retaliatory } \\
\hline 3. Delayed Aggression - Non- & 1.18 & 0.22 & $1.00-1.75$ & $.92^{* *}$ & $.77^{\star *}$ & & & & \\
\hline \multicolumn{10}{|l|}{ Retaliatory } \\
\hline 4. Self-Control & 3.55 & 0.88 & $2.00-5.40$ & $.29^{*}$ & .22 & $.36^{\star *}$ & & & \\
\hline 5. Trait Physical Aggression & 3.14 & 1.54 & $1.00-6.33$ & .18 & .18 & .16 & -.11 & & \\
\hline 6. Age & 18.98 & 1.07 & $18.00-22.00$ & -.10 & -.11 & -.08 & -.14 & .00 & \\
\hline 7. Gender & $n / a$ & $n / a$ & $n / a$ & .12 & .09 & .14 & -.05 & $.27^{*}$ & -.08 \\
\hline
\end{tabular}




\section{Whole-Brain Univariate Analyses}

Across the entire brain, retaliatory aggression events (as compared to nonretaliatory aggression events), were associated with large bilateral clusters that each included the anterior to posterior insula, the temporoparietal junction, the superior temporal sulcus, and the dorsal striatum (caudate, globus pallidus, putamen; Supplemental Figure 1; Supplemental Table 2).

\section{Neural Correlates of Behavior: VMPFC Correlates of Intertemporal Aggression}

To correlate decision behavior on the InterTemporal Aggression Paradigm with neural activity during such decisions, we selected a sub-sample of participants who had made enough of each decision type (i.e., delayed, immediate) to yield reliable BOLD estimates. Specifically, we decided to only analyze the data of participants who selected at least three instances of both delayed and immediate aggression, $N=35$ (22 females, 13 males; age: $M=18.89, S D=1.11$, range: $18-22)$. This cutoff of 3 was motivated by the need to exclude participants who did not make enough decisions to provide reliable BOLD estimates associated with those decisions, and conversely, by the desire to retain as many participants as possible in this analysis. We believe that our cutoff adequately balanced these competing constraints. Participants who were excluded from analyses according to this cutoff did not report different levels of trait physical aggression, $t(59)=-0.08, p=.941$, or trait self-control, $t(59)=1.69, p=.097$, than participants who passed this response cutoff.

Delayed versus immediate aggression was unassociated with any brain region using a whole-brain approach. Using an $\mathrm{ROI}$ approach, delayed aggression was 
associated with greater activity in the VMPFC (Figure 2A; 74 voxels; peak voxel: $Z=$

3.16, $p=.024, \mathrm{MNI}$ coordinates $[\mathrm{x}, \mathrm{y}, \mathrm{z}]=-4,62,-4)$.

Figure 2. (A) VMPFC activity associated with a preference for delayed-greater > immediate-lesser aggression. Green voxels depict VMPFC ROI mask. (B) Brain regions showing reduced functional connectivity with the VMPFC during delayed aggression. (C) Brain regions showing reduced functional connectivity with the VMPFC during immediate aggression. Coordinates are in MNI space. Data are from the subset of 35 participants who provided sufficient variability in their intertemporal decisions.

A

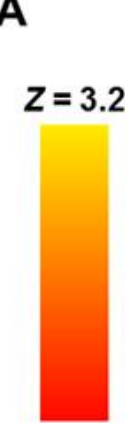

$$
Z=2.3
$$
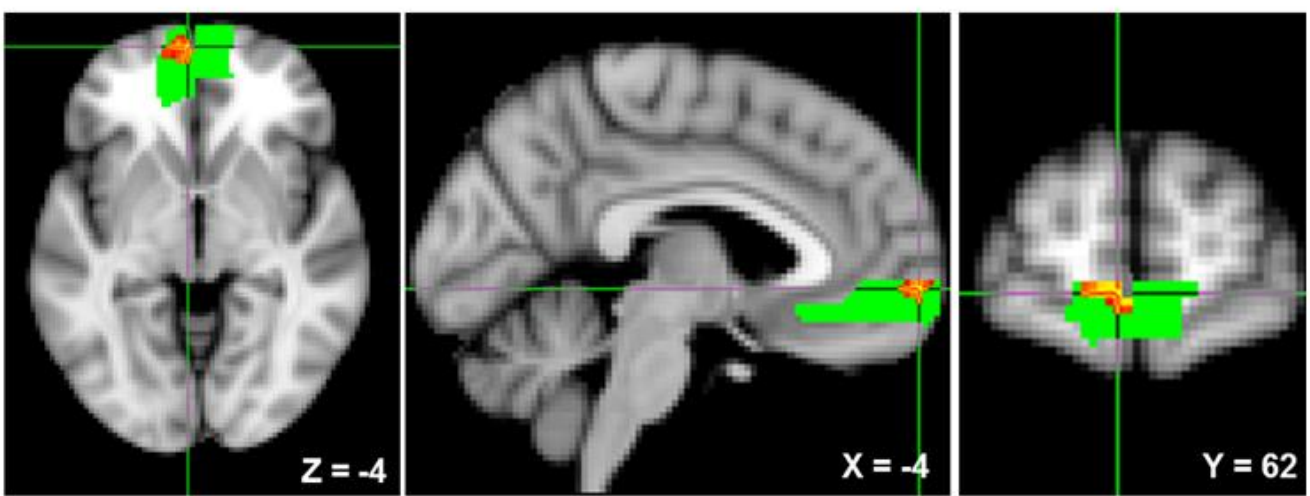

B
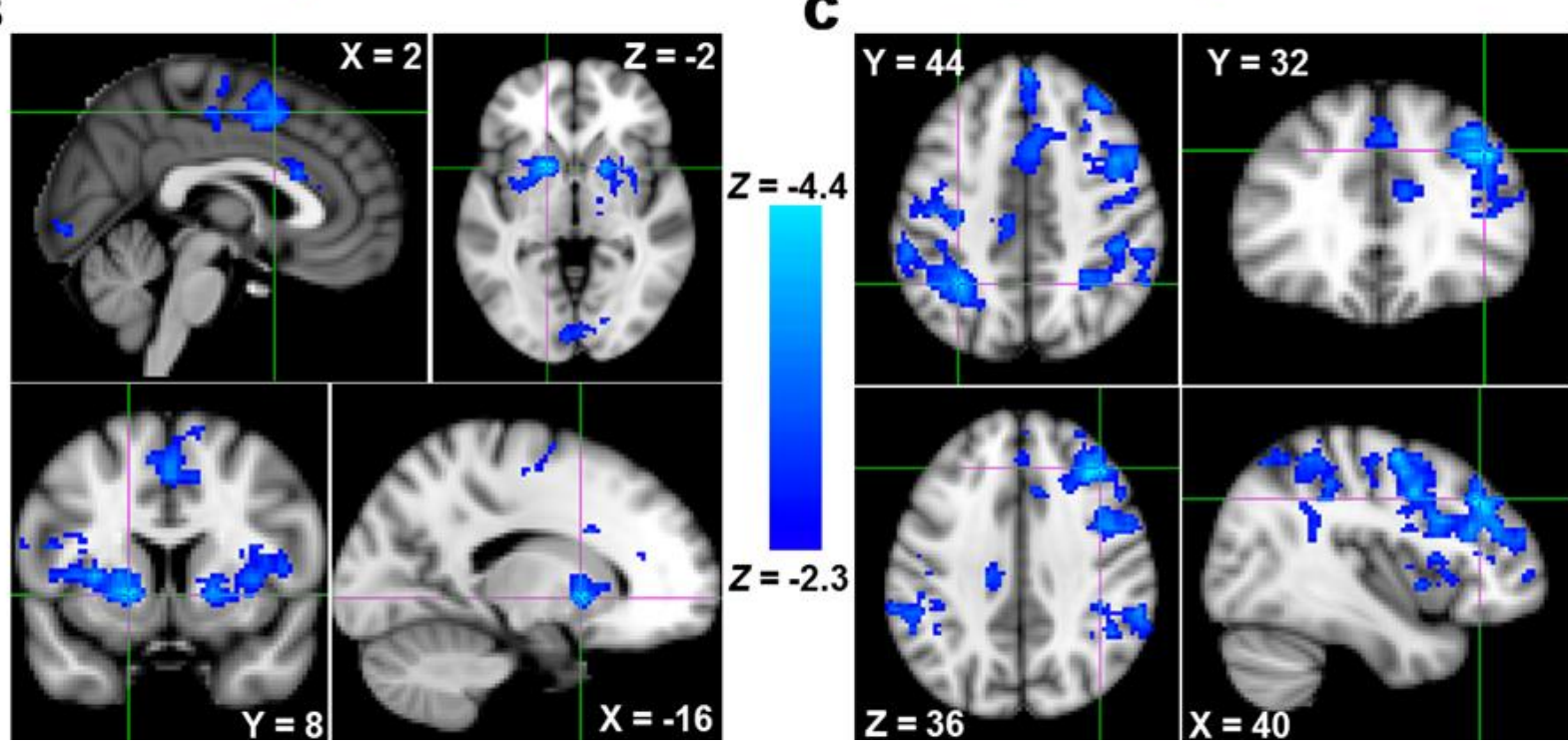


\section{Functional Connectivity Analyses}

Delayed aggression. Using the same subset of 35 participants, we observed that delayed aggression was associated with reduced functional connectivity (VMPFC $\mathrm{x}$ Delayed Aggression > Baseline) between the previously-observed VMPFC cluster and a range of regions that largely centered on the dorsal striatum, insula, and supplemental motor area (Figure 2B, Supplemental Table 3). No brain regions exhibited greater functional connectivity with the VMPFC during delayed aggression.

Immediate aggression. With an identical approach to the previous PPI analyses, we observed that immediate aggression was associated with reduced functional connectivity (VMPFC x Immediate Aggression > Baseline) between the VMPFC cluster and the frontoparietal network (dorsolateral PFC, superior parietal lobule) and the cingulo-opercular network (dorsal anterior cingulate cortex, ventrolateral prefrontal cortex; Figure 2C, Supplemental Table 4). No brain regions exhibited greater functional connectivity with the VMPFC during immediate aggression.

\section{VMPFC Correlations with Personality Traits}

Activity in the VMPFC cluster that was significantly associated with delayed aggression was associated with less trait physical aggression, $r(33)=-.36, p=.031$, yet was unassociated with self-control, $r(33)=.30, p=.082$.

\section{Indirect Effect Modeling}

To test for the presence of an indirect effect, whereby the association between trait physical aggression and delayed aggression was explained, in part, by VMPFC activation during intertemporal decision-making, we conducted an indirect effect analysis using the PROCESS macro v.3.1 for SPSS (all predictors mean-centered, 
model 4; 5,000 bias-corrected and accelerated bootstrapped re-samples; Hayes, 2012). Visual inspection of scatterplots of each path of the model did not suggest the presence of outlier effects (Figure 3A-C).

Figure 3. Scatterplots depicting zero-order correlations between key variables included in the mediation model. Data are from the subset of 35 participants who provided sufficient variability in their intertemporal decisions.
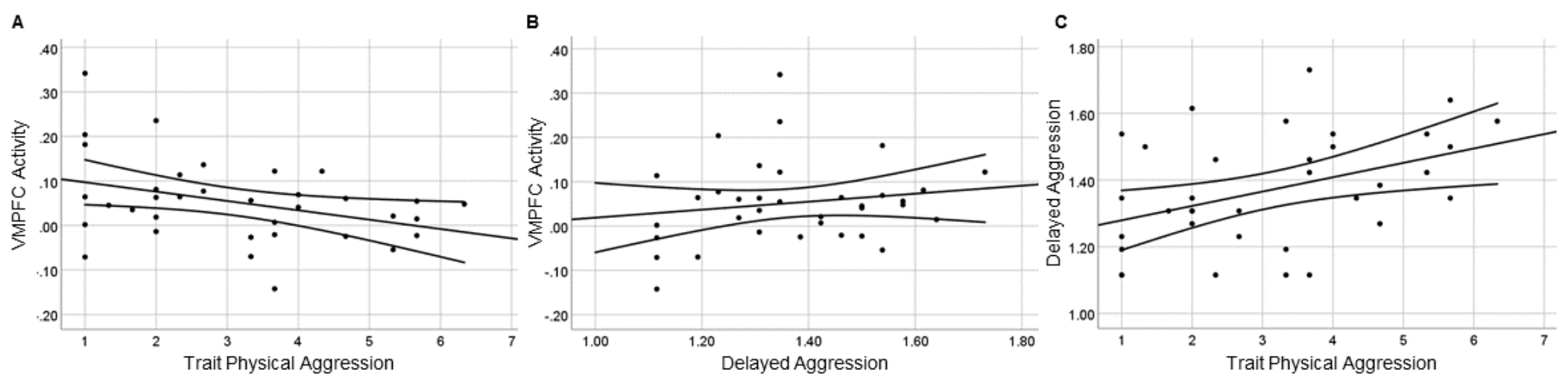

In this model, trait physical aggression was the predictor, VMPFC activity during delayed aggression was the 'mediator', and the intertemporal aggression index was the dependent variable. This model explained $38.19 \%$ of the variance in delayed aggressive behavior, $F(2,32)=6.33, M S E=0.02, p=.005, R^{2}=.28$. Doing so revealed a significant indirect effect, whereby VMPFC activity during delayed aggression explained a significant amount of the variance in the effect of trait physical aggression on delayed aggressive behavior, $B=-.13, S E=.07,95 \% C l=-.282,-.009$ (Figure 4). As indicators of statistical suppression, the direct effect of trait physical aggression on delayed aggressive behavior became stronger after controlling for the indirect effect of VMPFC activity, and the indirect effect was opposite in sign to the direct effect (see MacKinnon, Krull, \& Lockwood, 2000). 
Figure 4. An indirect effect in which dispositionally-aggressive individuals exhibited lesser VMPFC activity during intertemporal aggression decisions, which in turn was associated with more delayed aggressive behavior (across Retaliatory and NonRetaliatory conditions). Values represent standardized regression coefficients, parenthesized value is the direct effect after controlling for the indirect effect. Data are from the subset of 35 participants who provided sufficient variability in their intertemporal decisions. ${ }^{* *} p<.01,{ }^{*} p<.05$

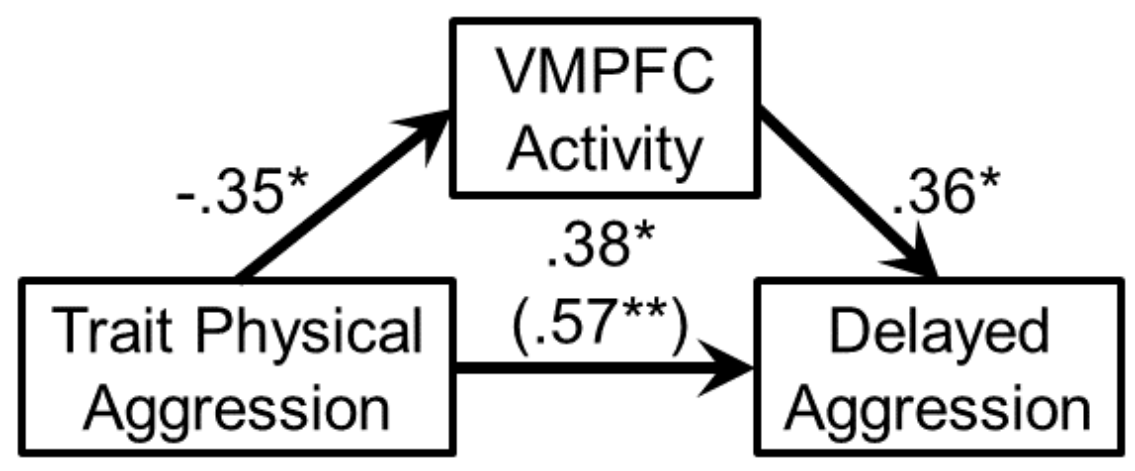

\section{Discussion}

Conventionally, aggression has been studied in a static temporal framework in which decisions about whether to be aggressive or not are dealt with purely in the 'here and now'. Recent work demonstrating the core feature of reward in aggressive behavior implies that aggression is a rewarding behavior that would greatly benefit from an intertemporal framework (Chester, 2017). To study this potential intertemporal nature of aggression, we modified a well-validated aggression paradigm to allow participants to decide between inflicting a smaller amount of aggression immediately or a considerably larger amount of aggression after a delay. We combined this novel paradigm with functional neuroimaging and personality trait assessment to attempt to understand the neural mechanisms and trait predictors of delayed versus immediate aggression. 
Data from our behavioral task revealed that 26 of our 61 participants selected the immediate-yet-lesser aggression option across $92-100 \%$ of the trials of the task. This tendency may reflect a desire to inflict a lesser amount of harm upon participants' opponents, but it may also reflect the well-established tendency for people to select an immediate reward, rather than enduring a delay for a longer-term goal (Mischel, 2014). Participants who tended to select immediate over delayed aggression did report less self-control than those who chose delayed rewards. Given that self-control is a wellestablished inhibitor of aggression (Denson, DeWall, \& Finkel, 2012), it is more likely that participants' bias towards the immediate option reflects a motivation to receive an immediate reward and not to reduce the harm that one has inflicted.

Provocation increased the amount of delayed-yet-more-severe aggression doled out in retaliation. This finding supports the idea that provoked individuals are willing to incur a delay, so long as it translates to a greater eventual punishment. However, it remains uncertain whether the preferences for immediate versus delayed preferences on the ITAP are durable across time and contexts. If aggression preferences on the ITAP are found to be robust, this would suggest that the task is tapping into the wellknown human tendencies towards reactive and proactive aggression (Raine et al., 2006). Future work should test whether preferences towards delayed aggression correspond to proactive aggression levels and if immediate aggression preferences correspond to reactive aggression levels.

Participants' bias towards delayed aggression was associated with greater activity in the VMPFC, a region reliably associated with the selection of other delayed rewards and self-regulation more generally (Frost \& McNaughton, 2017; Hare et al., 
2014). The VMPFC serves as an integrative hub that represents the ultimate value of an option after accounting for the various sources of potential value (Hare et al., 2014). This neural finding lends further evidence for the similarities between intertemporal choice in aggression and that in other reward-based domains. Functional connectivity analyses shed further light on these neural findings, revealing that delayed aggression was associated with reduced functional connectivity between the VMPFC and brain regions known to positively correlate with impulsive and reactive choices, among other behavioral outcomes (Dambacher et al., 2014; McClure et al., 2004, 2007). Conversely, immediate aggression was associated with less functional connectivity between the VMPFC and neural networks that subserve controlled self-regulation (Kelley, Wagner, \& Heatherton, 2015; Noël, Brevers, \& Bechara, 2013). These connectivity findings imply that immediate rewards are the result of not only reduced VMPFC activation but also less cross-talk between this brain region and broader cortical networks that serve to inhibit immediate impulses and allow for long-term goal pursuit.

Dispositionally-aggressive participants did not exhibit an explicit preference for delayed or immediate aggression. However, this trait was correlated with reduced VMPFC activity during such decisions, which explained substantial variance in the association between dispositional aggression and intertemporal preferences. This indirect effect implied that dispositionally-aggressive individuals' tendencies toward reactive aggression exist because these individuals fail to adequately recruit the prefrontal regulatory mechanisms necessary to inhibit their reactive aggression. Further evidence for this proposal came from the suppression effect we observed, in which VMPFC function served to blunt the preference for delayed aggression among 
dispositionally-aggressive individuals. Echoing other neural investigations, VMPFC dysfunction appears to promote reactive aggression and undermine tendencies towards delayed responses (Blair, 2016; Chester et al., 2017). Work that seeks to reduce reactive aggression should target interventions towards bolstering the regulatory functions of this region.

\section{Limitations and Future Directions}

There were many aspects of this present research that temper our conclusions and suggest avenues for future work. First, while some delay discounting paradigms employ fixed reward magnitude and/or temporal delay, others employ an adaptive task that calibrates the reward magnitude and/or temporal delay to participants' ideographic discounting curves (e.g., Steinberg et al., 2009). Because of the limited number of trials, we were unable to vary the magnitude of the two aggression options or the delay. Subsequent research will benefit from adopting the personally-calibrated task designs that have well-served the delay discounting literature. Second and third, our findings were correlational and relied on reverse inferences about what a given pattern of brain activation indicated about participants' underlying psychological processes. Future work could use brain stimulation approaches on the VMPFC to side-step both of these limitations.

Fourth, our undergraduate sample constrains our inferences and replication attempts are necessary with more diverse, aggressive, and impulsive populations. Fifth, the 'immediate aggression' option may not have reflected aggressive motivations at all, as participants may have felt compelled to select one of the options and prosociallyselected the option that inflicted the least amount of harm. A potential solution might be 
to create an experimental situation in which the participant is extremely motivated to aggress against their opponent, thus increasing the aggression exhibited on the task. Sixth, a large portion of our sample were unable to be included in analyses that contrasted delated versus immediate aggression because they selected only immediate options. The cutoff we used to determine who was included in this analysis was largely arbitrary, though it was motivated by two concerns: the desire to exclude participants with too few BOLD measurements of one type of aggression to yield reliable neural correlates, and the desire to retain as much of the original sample as possible in order to increase statistical power. Our threshold of three instances of each aggression-type was considered a reasonable compromise between these two concerns. Going forward, modifications to the task that promote a greater variability in response profiles will be needed.

Seventh, our small sample size increased the likelihood that we made observed both false positive and false negative results. As such, this study may have made conclusions based on spuriously-significant results and failed to observe effects that are, in fact, true. Future work with larger samples and a greater number of measurements is needed to establish the veridicality and replicability of our results. Finally, we were unable to establish the temporal sequence of variables in our indirect effect model because we employed a cross-sectional design (Preacher, 2015). As such, the presence of our proposed indirect effect cannot be conclusively established until future work examines these relationships in a longitudinal sequence that allows for the articulation of the timeline of these effects.

\section{Conclusions}


Why do some people lash out at whatever provokes them and others are able to bide their time and exact revenge when it is most harmful? Aggression is rarely studied in this intertemporal context and we sought to fill this gap in the literature. Participants who preferred delayed yet more severe forms of aggression, over immediate but less severe forms, exhibited psychological and neural indicators of greater self-control. As such, delayed aggression is a curious specimen, in which greater harm infliction is a product of successful self-control, not a failure thereof. 


\section{References}

Berkman, E. T., Livingston, J. L., \& Kahn, L. E. (2017). Finding the "self" in selfregulation: The identity-value model. Psychological Inquiry, 26(2), 77-98. doi:10.1080/1047840X.2017.1323463

Berns, G. S., Laibson, D., \& Loewenstein, G. (2007). Intertemporal choice-toward an integrative framework. Trends in Cognitive Sciences, 11(11), 482-488. doi:10.1016/j.tics.2007.08.011

Blair, R. J. R. (2010). Neuroimaging of psychopathy and antisocial behavior: a targeted review. Current Psychiatry Reports, 12(1), 76-82. doi:10.1007/s11920-009-0086$\mathrm{X}$

Blair, R. J. (2016). The neurobiology of impulsive aggression. Journal of Child and Adolescent Psychopharmacology, 26(1), 4-9. doi:10.1016/S0193$953 \times(05) 70319-1$

Bushman, B. J., \& Anderson, C. A. (2001). Is it time to pull the plug on hostile versus instrumental aggression dichotomy? Psychological Review, 108(1), 273-279. doi:10.1037/0033-295X.108.1.273

Cherek, D. R., \& Lane, S. D. (1999). Laboratory and psychometric measurements of impulsivity among violent and nonviolent female parolees. Biological Psychiatry, 46(2), 273-280. doi:10.1016/S0006-3223(98)00309-6

Cherek, D. R., Moeller, F. G., Dougherty, D. M., \& Rhoades, H. (1997). Studies of violent and nonviolent male parolees: I. Laboratory and psychometric measurements of impulsivity. Biological Psychiatry, 41(5), 523-529. doi:10.1016/S0006-3223(96)00059-5 
Chester, D. S. (2017). The role of positive affect in aggression. Current Directions in Psychological Science, 26(4), 366-370. doi:10.1177\%2F0963721417700457

Chester, D. S. \& DeWall, C. N. (2016). The pleasure of revenge: Retaliatory aggression arises from a neural imbalance toward reward. Social Cognitive and Affective Neuroscience, 11(7), 1173-1182. doi:10.1093/scan/nsv082

Chester, D. S. \& DeWall, C. N. (2017). Combating the sting of rejection with the pleasure of revenge: A new look at how emotion shapes aggression. Journal of Personality and Social Psychology, 112(3), 413-430. doi:10.1037/pspi0000080

Chester, D. S. \& DeWall, C. N. (2018). Personality correlates of revenge-seeking: Multidimensional links to physical aggression, impulsivity, and aggressive pleasure. Aggressive Behavior, 44(3), 235-245. doi:10.1002/ab.21746

Chester, D. S., Lynam, D. R., Milich, R., \& DeWall, C. N. (2017). Physical aggressiveness and gray matter deficits in ventromedial prefrontal cortex. Cortex, 97(1), 17-22. doi:10.1016/j.cortex.2017.09.024

Chester, D. S., Lynam, D. R., Milich, R., \& DeWall, C. N. (2018). Neural mechanisms of the rejection-aggression link. Social Cognitive and Affective Neuroscience, 13(5), 501-512. doi:10.1093/scan/nsy025

da Matta, A., Gonçalves, F. L., \& Bizarro, L. (2012). Delay discounting: Concepts and measures. Psychology \& Neuroscience, 5(2), 135-146. doi:10.3922/j.psns.2012.2.03

Dambacher, F., Sack, A. T., Lobbestael, J., Arntz, A., Brugman, S., \& Schuhmann, T. (2014). Out of control: Evidence for anterior insula involvement in motor 
impulsivity and reactive aggression. Social Cognitive and Affective

Neuroscience, 10(4), 508-516. doi:10.1093/scan/nsu077

Denson, T. F., DeWall, C. N., \& Finkel, E. J. (2012). Self-control and aggression. Current Directions in Psychological Science, 21(1), 20-25. doi:10.1177\%2F0963721411429451

Denson, T. F., Pedersen, W. C., \& Miller, N. (2006). The displaced aggression questionnaire. Journal of Personality and Social Psychology, 90(6), 1032-1051. doi:10.1037/0022-3514.90.6.1032

Frost, R., \& McNaughton, N. (2017). The neural basis of delay discounting: A review and preliminary model. Neuroscience and Biobehavioral Reviews, 79(1), 48-65. doi:10.1016/j.neubiorev.2017.04.022

Hare, T. A., Camerer, C. F., \& Rangel, A. (2009). Self-control in decision-making involves modulation of the vmPFC valuation system. Science, 324(5927), 646648. doi:10.1126/science. 1168450

Hare, T. A., Hakimi, S., \& Rangel, A. (2014). Activity in dIPFC and its effective connectivity to VmPFC are associated with temporal discounting. Frontiers in Neuroscience, 8(1), 50-50. doi:10.3389/fnins.2014.00050

Hayes, A. F. (2012). PROCESS: A Versatile Computational Tool for Observed Variable Mediation, Moderation, and Conditional Process Modeling (Version 2.0) [Software]. Available from http://www.afhayes.com/public/process2012.pdf Heller, R., Stanley, D., Yekutieli, D., Rubin, N., \& Benjamini, Y. (2006). Cluster-based analysis of fMRI data. Neurolmage, 33(2), 599-608. doi:10.1016/j.neuroimage.2006.04.233 
Kelley, W. M., Wagner, D. D., \& Heatherton, T. F. (2015). In search of a human selfregulation system. Annual review of Neuroscience, 38(1), 389-411. doi:10.1146/annurev-neuro-071013-014243

Kirby, K. N., Petry, N. M., \& Bickel, W. K. (1999). Heroin addicts have higher discount rates for delayed rewards than non-drug-using controls. Journal of Experimental Psychology: General, 128(1), 78-87/ doi:10.1037//0096-3445.128.1.78

Koepfler, J., Brewster, J., Stoloff, M., \& Saville, B. (2012). Predicting police aggression: Comparing traditional and non-traditional prediction models. Journal of Police and Criminal Psychology, 27(2), 141-149. doi:10.1007/s11896-012-9101-y

Krämer, U. M., Jansma, H., Tempelmann, C., \& Münte, T. F. (2007). Tit-for-tat: The neural basis of reactive aggression. Neurolmage, 38(1), 203-211. doi:10.1016/j.neuroimage.2007.07.029

Levy, D. J., \& Glimcher, P. W. (2012). The root of all value: a neural common currency for choice. Current Opinion in Neurobiology, 22(6), 1027-1038. doi:10.1016/j.conb.2012.06.001

Loewenstein, G. F. (1988). Frames of mind in intertemporal choice. Management Science, 34(2), 200-214. doi:10.1287/mnsc.34.2.200

MacKinnon, D. P., Krull, J. L., \& Lockwood, C. M. (2000). Equivalence of the mediation, confounding, and suppression effect. Prevention Science, 1(4), 173-181. doi:10.1023/A:1026595011371

McClure, S. M., Ericson, K. M., Laibson, D. I., Loewenstein, G., \& Cohen, J. D. (2007). Time discounting for primary rewards. Journal of Neuroscience, 27(21), 57965804. doi:10.1523/JNEUROSCI.4246-06.2007 
McClure, S. M., Laibson, D. I., Loewenstein, G., \& Cohen, J. D. (2004). Separate neural systems value immediate and delayed monetary rewards. Science, 306(5695), 503-507. doi:10.1126/science.1100907

Miller, J. D., \& Lynam, D. R. (2003). Psychopathy and the five-factor model of personality: A replication and extension. Journal of Personality Assessment, 81(2), 168-178. doi:10.1207/S15327752JPA8102_08

Mischel, W. (2014). The marshmallow test: understanding self-control and how to master it. New York: Random House. [no doi available]

Moore, S. C., \& Foreman-Peck, J. (2009). Alcohol consumption predicts violent victimisation, impulsive decision making predicts violence. The Open Behavioral Science Journal, 3(1), 28-33. [no doi available]

Noël, X., Brevers, D., \& Bechara, A. (2013). A neurocognitive approach to understanding the neurobiology of addiction. Current Opinion in Neurobiology, 23(4), 632-638. doi:10.1016/j.conb.2013.01.018

Preacher, K. J. (2015). Advances in mediation analysis: A survey and synthesis of new developments. Annual Review of Psychology, 66(1), 825-852. doi:10.1146/annurev-psych-010814-015258

Raine, A., Dodge, K., Loeber, R., Gatzke-Kopp, L., Lynam, D., Reynolds, C., ... \& Liu, J. (2006). The reactive-proactive aggression questionnaire: Differential correlates of reactive and proactive aggression in adolescent boys. Aggressive Behavior, 32(2), 159-171. doi:10.1002/ab.20115 
Reynolds, B. (2006). A review of delay-discounting research with humans: relations to drug use and gambling. Behavioural Pharmacology, 17(8), 651-667. doi:10.1097/FBP.0b013e3280115f99

Smith, S. M., Jenkinson, M., Woolrich, M. W., Beckmann, C. F., Behrens, T. E. J., Johansen-Berg, H., ... Matthews, P. M. (2004). Advances in functional and structural MR image analysis and implementation as FSL. Neurolmage, 23(1), S208-S219. doi:10.1016/j.neuroimage.2004.07.051

Steinberg, L., Graham, S., O'Brien, L., Woolard, J., Cauffman, E., \& Banich, M. (2009). Age differences in future orientation and delay discounting. Child Development, 80(1), 28-44. doi:10.1111/j.1467-8624.2008.01244.x

Tangney, J. P., Baumeister, R. F., \& Boone, A. L. (2004). High self-control predicts good adjustment, less pathology, better grades and interpersonal success. Journal of Personality, 72 (2), 271-324. doi:10.1111/j.0022-3506.2004.00263.x

Taylor, S. P. (1967). Aggressive behavior and physiological arousal as a function of provocation and the tendency to inhibit aggression. Journal of Personality, 35(2), 297-310. doi:10.1111/j.1467-6494.1967.tb01430.x

Tzourio-Mazoyer, N., Landeau, B., Papathanassiou, D., Crivello, F., Etard, O., Delcroix, N., ... \& Joliot, M. (2002). Automated anatomical labeling of activations in SPM using a macroscopic anatomical parcellation of the MNI MRI single-subject brain. Neurolmage, 15(1), 273-289. doi:10.1006/nimg.2001.0978

Webster, G. D., DeWall, C. N., Pond, R. S., Deckman, T., Jonason, P. K., Le, B. M., ... Bator, R. J. (2014). The brief aggression questionnaire: psychometric and 
behavioral evidence for an efficient measure of trait aggression. Aggressive Behavior, 40(2), 120-139. doi:10.1002/ab.21507

Woolrich, M. W., Jbabdi, S., Patenaude, B., Chappell, M., Makni, S., Behrens, T., ... Smith, S. M. (2009). Bayesian analysis of neuroimaging data in FSL. Neurolmage, 45(1), S173-S186. doi:10.1016/j.neuroimage.2008.10.055

Worsley, K. J. (2001). Statistical analysis of activation images. Functional MRI: An Introduction to Methods, 14(1), 251-270. [no doi available]

Zhao, J., Kirwen, N., Johnson, J., \& Vigo, R. (2017). Anger and intertemporal choice: The behavioral approach system and the interactive effects of trait and state anger. Personality and Individual Differences, 110, 60-64.

doi:10.1016/j.paid.2017.01.022 
Supplemental Figure 1. Brain regions associated with Retaliatory > Non-Retaliatory Aggression. Coordinates are in MNI space.
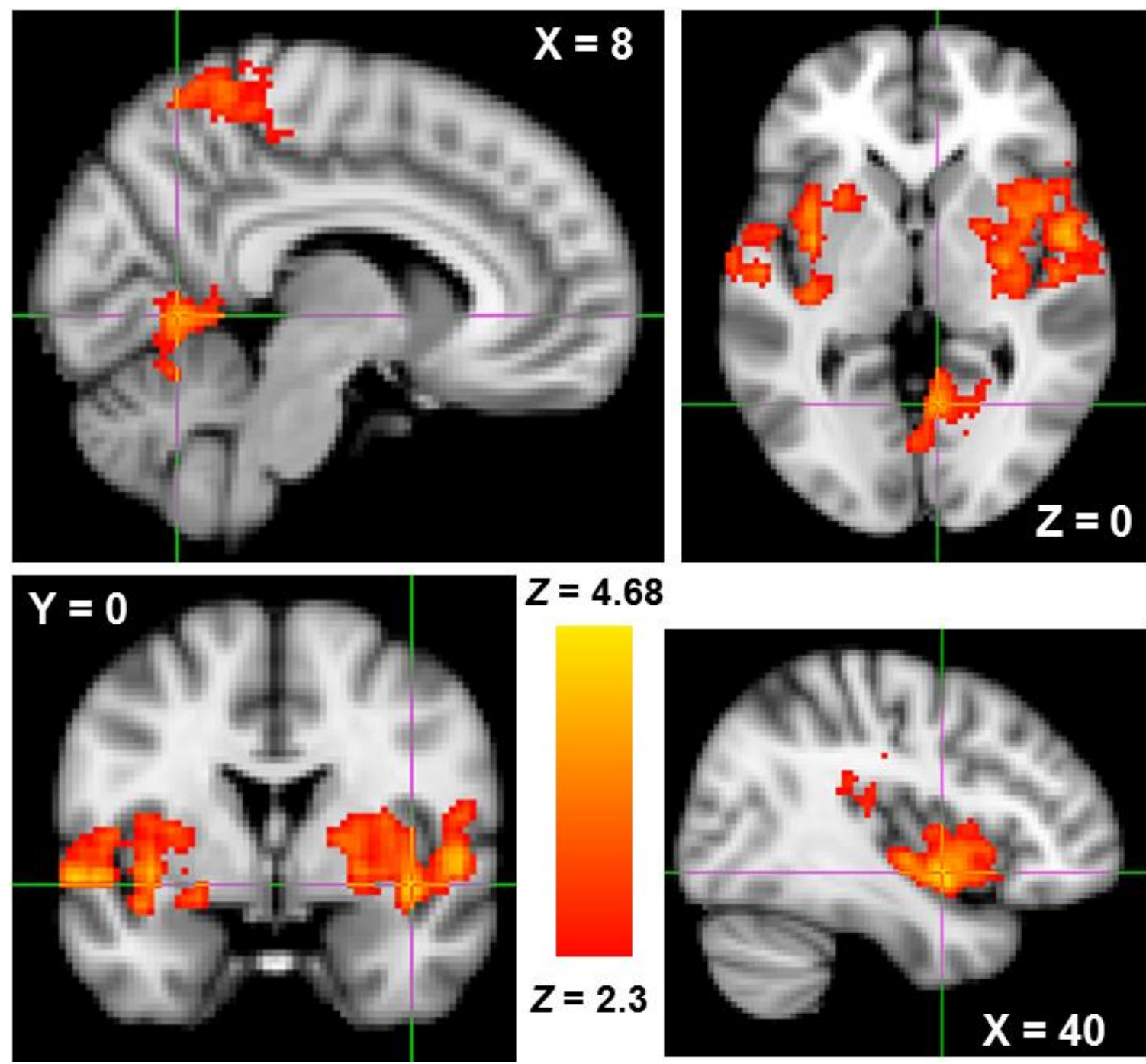

$Z=4.68$
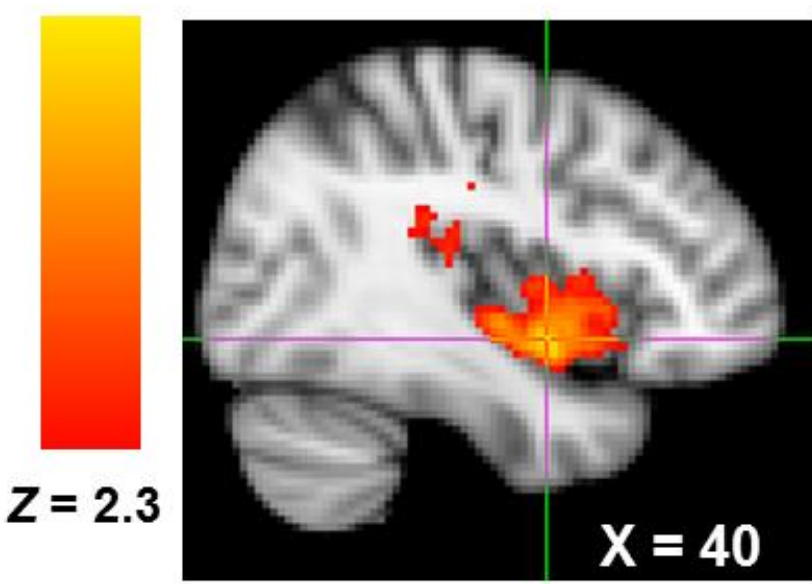
Supplemental Table 1. Temporal order of the Retaliatory versus Non-Retaliatory trials of the InterTemporal Aggression Paradigm.

\begin{tabular}{ll}
\hline Trial & Type of Trial \\
\hline 1 & Non-Retaliatory \\
2 & Retaliatory \\
3 & Retaliatory \\
4 & Non-Retaliatory \\
5 & Retaliatory \\
6 & Retaliatory \\
7 & Non-Retaliatory \\
8 & Retaliatory \\
9 & Retaliatory \\
10 & Non-Retaliatory \\
11 & Non-Retaliatory \\
12 & Non-Retaliatory \\
13 & Non-Retaliatory \\
14 & Non-Retaliatory \\
15 & Non-Retaliatory \\
16 & Non-Retaliatory \\
17 & Retaliatory \\
18 & Retaliatory \\
19 & Retaliatory \\
20 & Retaliatory \\
21 & Non-Retaliatory \\
22 & Retaliatory \\
23 & Retaliatory \\
24 & Non-Retaliatory \\
25 & Retaliatory \\
26 & Retaliatory \\
\hline &
\end{tabular}


Supplemental Table 2. Whole-brain fMRI main effect results from the Retaliatory > NonRetaliatory Aggression trials contrast. STS = superior temporal sulcus, TPJ = temporoparietal junction.

\begin{tabular}{lllll}
\hline \multirow{2}{*}{ Cluster } & Brain Region & Voxels & Peak $Z$ & coordinates $(x, y, z)$ \\
\hline 1 & Dorsal Striatum, Insula, STS, TPJ & 4,212 & 4.39 & $40,0,-8$ \\
2 & Dorsal Striatum, Insula, STS, TPJ & 3,136 & 4.68 & $-36,-4,-4$ \\
3 & Superior Parietal Lobule & 1,092 & 3.79 & $12,-50,66$ \\
4 & Cerebellum, Occipital Lobe & 1,076 & 4.12 & $8,-60,0$ \\
\hline
\end{tabular}


Supplemental Table 3. Brain regions showing reduced functional connectivity with the VMPFC during delayed aggression.

\section{Peak MNI}

\begin{tabular}{lllll} 
Cluster & Brain Region & Voxels & Peak $Z$ & coordinates $(x, y, z)$ \\
\hline 1 & Dorsal Striatum, Insula & 1,105 & -4.49 & $-16,8,-2$ \\
2 & Dorsal Striatum, Insula, Thalamus & 1,018 & -3.89 & $18,8,-2$ \\
3 & Supplemental Motor Area & 930 & -3.60 & $2,10,50$ \\
4 & Mid-Cingulate Cortex & 401 & -3.80 & $-10,14,28$ \\
5 & Occipital Lobe & 243 & -3.60 & $-6,-90,0$
\end{tabular}


Supplemental Table 4. Brain regions showing reduced functional connectivity with the VMPFC during immediate aggression. $D L P F C=$ dorsolateral prefrontal cortex, DMPFC $=$ dorsomedial prefrontal cortex, TPJ $=$ temporoparietal junction, $V L P F C=$ ventrolateral prefrontal cortex

Peak MNI coordinates

Cluster Brain Region $\quad$ Voxels Peak $Z \quad(x, y, z)$

Post-Central Gyrus, Superior

$1 \quad$ Parietal Lobule, TPJ $\quad 5,438 \quad 4.28 \quad-32,-52,44$

2 DLPFC, DMPFC, Insula, Pre-

Central Gyrus, VLPFC $\quad 5,065 \quad 4.36 \quad 40,32,36$

3 Post-Central Gyrus, Superior

Parietal Lobule, TPJ $\quad 1,511 \quad 3.82 \quad 50,-42,22$ 